\title{
Awareness, Attitude and Use of Chlorhexidine Gel for Cord Care in a Well-Baby Clinic in Port Harcourt, Nigeria
}

\author{
Josephine Enekole Aitafo ${ }^{1}$, Boma Awoala West $^{2}$, Tamunoiyowuna Grace Okari ${ }^{3}$ \\ ${ }^{1}$ Department of Paediatrics, Rivers State University Teaching Hospital, Nigeria \\ ${ }^{2,3}$ Department of Paediatrics and Child Health, Rivers State University, Nkpolu, Port Harcourt, Nigeria
}

Corresponding Author: Josephine Enekole Aitafo

\begin{abstract}
Introduction: Neonatal mortality rate in Nigeria is high and sepsis is a significant contributor. The World Health Organization recommends the use of $7.1 \%$ chlorhexidine gel for cord care to prevent neonatal sepsis and mortality.

Aim: The study aimed to assess the awareness, attitude and use of chlorhexidine gel for cord care among mothers in Port Harcourt.

Methods: A cross sectional study was carried out in the well-baby clinic in Rivers State University Teaching Hospital for 3 months. A research proforma with questions to assess awareness, attitude and use of chlorhexidine gel for cord care practices among the participants was used to collect data and analyzed with SPSS version 23. A score of $70 \%$ or more was taken as benchmark for good awareness, attitude and use.

Result: A total of 199 mothers participated in the study, 126(63.3\%) had heard of chlorhexidine gel, mostly from antenatal clinics. Thirty (23.8\%) knew that it prevents neonatal infections, $74(58.7 \%)$ perceived it was effective, but only $19(9.6 \%)$ used it. About a third of the mothers had good awareness and attitude towards the use of chlorhexidine gel but only 18(9\%) used it correctly. Mothers with tertiary level of education significantly had good attitude towards its use and poor knowledge correlated significantly with poor use, $\mathrm{P}<0.05$.

Conclusion: The level of awareness, attitude and use of CHX in cord care practices in Port Harcourt is poor. There is an urgent need to educate mothers on the proper use and benefits of chlorhexidine gel in cord care practices.
\end{abstract}

Keywords: Chlorhexidine gel, Cord Care, Awareness, Attitude, Use, Port Harcourt.

\section{INTRODUCTION}

Neonatal mortality (NNM) rates in developing countries especially in sub Saharan Africa where Nigeria is located is high. ${ }^{1}$ The neonatal mortality rate in Nigeria as at 2019 still remains high at 39/1000 live births NDHS with preterm deliveries, perinatal asphyxia and neonatal sepsis as leading causes. ${ }^{2}$

Neonates are at risk of developing sepsis when microorganisms gain entrance to their blood by colonizing their bodies and the umbilical cord stump, causing omphalitis. The common pathogenic microbes implicated in this infection include Staphylococcus aureus, group A Streptococcus, Escherichia coli, Klebsiella pneumoniae and Proteus mirabilis. ${ }^{3}$

In order to reduce the risk of neonatal sepsis (NNS) occurring from infected umbilical cord stump, appropriate cord care practices are advocated. These practices as advocated by the World Health Organization (WHO) include the use of sterile instrument in ligating the cord at birth, in addition to air drying of the 
umbilical stump as appropriate, provided that the delivery of the child took place under hygienic conditions. ${ }^{4,5}$ This is however not the case in sub-Saharan Africa, where a great number of deliveries are still taking place at home under unhygienic conditions, in addition to the application of harmful contaminated substances like soil, cow dung and toothpaste to the umbilical stump. ${ }^{6-10}$

In a bid to eliminate these unhealthy cord care practices, the WHO approved the use of an antiseptic and antimicrobial agent; $7.1 \%$ chlorhexidine for cord care especially in countries with neonatal mortality rates above 30 per 1000 live births as seen in Nigeria. ${ }^{2,411}$ This should be applied to the umbilical stump and base of the cord immediately after cutting the cord and application continued in the first week of life. Caregivers are expected to wash their hands before and after the application of chlorhexidine gel. ${ }^{4,11,12}$ Studies have shown that the application of $7.1 \%$ chlorhexidine gel within the first 24hours of life reduces the risk of omphalitis, neonatal sepsis and neonatal mortality. ${ }^{13,14,15}$

In 2012, the Federal Ministry of Health in Nigeria in partnership with the United States Agency for International Development (USAID) implemented the use of $7.1 \%$ chlorhexidine gel in the country and the pilot scheme was commenced in Bauchi and Sokoto States, ${ }^{16}$ with a program put in place to scale up its use nationally. The strategies adopted included creating awareness and demand for chlorhexidine gel use among others. Unfortunately, one of the key hindrances identified in the scale up program nationally was low awareness level of this new product among mothers and caregivers and the competitiveness of available alternative harmful products used for cord care in the communities. These challenges were expected to be overcome by educating health care workers and caregivers in the appropriate use of chlorhexidine gel. ${ }^{16}$

A review of the scheme over a 3year period in the pilot states showed a gradual increase in the use of CHX in both states from $0.7 \%$ in 2012 to $21.5 \%$ in 2015 in Bauchi State and from $0.8 \%$ to $17.1 \%$ between 2012 and 2015 in Sokoto State, Nigeria. ${ }^{17}$ These increases were however mostly derived from deliveries that took place in health facilities and not from the majority of the deliveries which took place in maternities and at home. ${ }^{17,18}$

Studies to assess the awareness level and use of chlorhexidine gel for cord care in Nigeria are very few. However, in a study conducted in Jos metropolis, Nigeria, the use of chlorhexidine gel in cord care was very poor at $6.9 \%, 5$ years after its introduction in Nigeria. ${ }^{19}$ No study has been carried out in Port Harcourt, Nigeria, ${ }^{7}$ years after the introduction of this policy. Hence the need to carry out this study in Port Harcourt in order to determine the awareness, attitude and the practice of using chlorhexidine gel for cord care in Port Harcourt.

\section{MATERIALS AND METHODS Study Design}

This study was a cross-sectional study carried out in the well-baby clinic over 3 months from 1st October, 2020 to 31st December, 2020.

\section{Study Site}

Rivers State University Teaching Hospital is a state-owned tertiary hospital located in the capital city of Port Harcourt. It is a 375 bedded hospital consisting of clinical and non- clinical departments. The clinical departments comprise of the departments of Paediatrics, Obstetrics and Gynaecology, Surgery, Internal medicine, Community medicine, Family medicine, Pharmacy, Nursing among others. It serves as a referral centre for all the government owned Primary Health Care facilities, general hospitals and private health facilities in the state as well as neighbouring states.

The well-baby clinic also known as the immunization clinic which sees an average of 25 babies daily, is run by the Community medicine department of the 
hospital and operates on Mondays to Fridays, from $8 \mathrm{am}$ to $2 \mathrm{pm}$.

\section{Study population}

The study population was made of all mothers attending the well-baby clinic.

\section{Sampling Size}

A convenient sampling size of 199 mother-baby pair was recruited into the study.

\section{Inclusion Criteria}

Mothers who gave informed consent and whose babies were 28 days to 24 months were consecutively recruited into the study.

\section{Exclusion Criteria}

Mothers who did not give informed consent and whose babies were either less than 28days old or greater than 24months were excluded from the study.

\section{Study Procedure}

A research team was constituted prior to commencement of the study, made up of the researchers and research assistants (house officers). Training was carried out by the lead researcher on proper administration of the pre-tested and validated questionnaire. COVID-19 safety protocols were strictly adhered to with research assistants and participants wearing face mask, regular use of hand sanitizers, maintenance of physical distancing and the provision of individual writing pens which were not retrieved thereafter.

The research Proforma was administered to each mother that was recruited. The socio-demographic characteristics obtained were age of mothers, mothers' level of education, mother's occupation, fathers' level of education, fathers' occupation and age of index child. Questions to assess mother's awareness, attitude and use of chlorhexidine gel were also asked and scores allotted to each response. A correct answer was assigned 1 point while an incorrect or 'I don't know' was assigned zero. Eight points were allocated to the section on mother's awareness. Mothers were determined to have good awareness of chlorhexidine gel if they had at least $70 \%$ and above (5-8) correct responses to the questions in the section on awareness. Awareness was said to be poor if the correct responses were less than $70 \%$ ( $<5$ correct responses). Three points were allocated to the section on attitude. Mothers were said to have good attitude if they had at least $70 \%$ and above (2-3) positive responses to the questions, and poor attitude if the positive responses were less than $70 \%(<2)$ in this section. Eight points were allocated to the section on the use of chlorhexidine gel. The use of CHX was said to be good if the mothers had $70 \%$ or more (5-8) positive responses and poor if the positive responses to questions in this section were less than $70 \%(<5)$.

\section{Ethical Clearance}

Ethical clearance was obtained from the Rivers State University Teaching Hospital Ethics Committee (REC number: RSUTH/REC/2021047). The proposed study was explicitly explained to the mothers attending the well-baby clinic and informed consent was obtained before the questionnaire was administered.

\section{Statistical Analysis}

All the information obtained were entered into an excel spreadsheet and data analyzed using SPSS version 23. Data was presented as frequency and percentages for categorical variables. Assessment of awareness, attitude and use of CHX gel was carried out by scoring each item selected, summation of the selected items, converting the summed scores to percentages and graded into good or poor, depending on the score of the participants.

Fishers' exact test was used to test for statistical significance in the associations between awareness, attitude and use, respectively and with socio-demographic features of participants. Statistical significance was set at $\mathrm{P}$ value $<0.05$. 
Logistic regression was introduced to test the strength of association between awareness, attitude and with use of CHX gel. Odd ratio was reported alongside its $95 \%$ confidence interval.

\section{RESULT}

\section{Awareness of Chlorhexidine gel}

Of 199 mothers recruited, majority $126(63.3 \%)$ had heard of chlorhexidine gel with the antenatal clinic $103(81.7 \%)$ being the commonest source of information. All $126(100 \%)$ mothers who had heard of CHX, knew it was used for cord care and should be applied on the cord. Forty-two (33.3\%) mothers knew chlorhexidine gel was applied once while the commonest benefit of chlorhexidine gel identified by the mothers was prevention of infection, 30(23.8\%). The commonest disadvantage of chlorhexidine gel recalled was delayed cord detachment, 23(18.3\%), Table I

Sixty-eight (34.2\%) respondents were assessed to have good awareness level with $70 \%$ or more scores while $131(65.8 \%)$ had poor awareness with less than $70 \%$ of the total awareness scores.

Table I: Mother's Awareness of Chlorhexidine gel

\begin{tabular}{|lc|}
\hline Variables & Frequency (\%) \\
\hline Have you heard of chlorhexidine gel? n=199 & $126(63.3)$ \\
Yes & $73(36.7)$ \\
No & $103(81.7)$ \\
Source of information of chlorhexidine gel? n=126 & \\
$\quad$ Antenatal clinic & $15(11.9)$ \\
Other health workers & $5(4.0)$ \\
Relatives & $3(2.4)$ \\
Neighbours/friends & \\
Is chlorhexidine gel used alone? n=126 & $42(33.3)$ \\
Yes & $54(42.9)$ \\
No & $30(23.8)$ \\
I don't know & \\
What are the benefits of chlorhexidine gel? & \\
n=126 & $30(23.8)$ \\
Prevents infection & $24(19.1)$ \\
Heals cord fast & $15(11.9)$ \\
Keeps cord clean & $57(45.2)$ \\
I don't know & \\
What are the disadvantages of chlorhexidine gel? & \\
n=126 & $23(18.2)$ \\
Delayed cord detachment & $2(1.6)$ \\
Expensive & $7(5.6)$ \\
Cause rash/bleeding & $94(74.6)$ \\
I don't know & \\
\hline
\end{tabular}

\section{Attitude of mothers towards the use of chlorhexidine gel}

Of 126 respondents who have heard of chlorhexidine gel, 74(58.7\%) believed it is effective, 62(49.2\%) would use it again and $75(59.5 \%)$ would recommend it.

Seventy-one $(56.3 \%)$ mothers were assessed to have good attitude while $55(43.7 \%)$ had poor attitude, Table II.

Table II: Attitude of mothers towards the use of chlorhexidine gel

\begin{tabular}{|lc|}
\hline Variables & $\begin{array}{c}\text { Frequency, } \\
\text { n=126 (\%) }\end{array}$ \\
\hline $\begin{array}{l}\text { Do you believe chlorhexidine gel is effective? } \\
\text { Yes }\end{array}$ & $74(58.7)$ \\
No & $52(41.3)$ \\
Would you use it again? & \\
Yes & $62(49.2)$ \\
No & $64(50.8)$ \\
Would you recommend chlorhexidine gel? & \\
Yes & $75(59.5)$ \\
No & $51(40.5)$ \\
\hline
\end{tabular}

\section{The use of chlorhexidine gel}

Most mothers, 191(96.0\%) took care of the umbilical cord of the index baby and methylated spirit $113(56.8 \%)$ was the commonest cord care agent used. Only 19(9.5\%) mothers used CHX for cord care with their index baby. Majority of mothers began cord care within the 1st 24hours of birth, 128(64.3\%). The choice of agent used for cord care was majorly influenced by information received from the antenatal clinic as seen in $120(60.3 \%)$ of mothers.

Chlorhexidine gel had been used in the past by only $67(33.7 \%)$ mothers. Of these 67 mothers, $16(23.9 \%)$ applied the gel once daily and application over the cord and surrounding skin was done by $36(53.7 \%)$ mothers. Forty-nine (73.1\%) mothers washed their hands always before application while 40(59.7\%) kept cord dry after application. Fifteen (22.4\%) mothers stopped application of the gel after cord detachment. Most 45(67.2\%) of their baby's cords were detached between 7-14 days of application of chlorhexidine gel. Only 16 mothers experienced side-effects with the use of chlorhexidine gel, with most $10(62.5 \%)$ attributing delayed cord detachment to the use of chlorhexidine gel, Table III.

The assessment of the proper use of CHX was said to be good in $18(9.0 \%)$ mothers and poor among 181(91.0\%) mothers. 
Josephine Enekole Aitafo et.al. Awareness, attitude and use of chlorhexidine gel for cord care in a well-baby clinic in Port Harcourt, Nigeria.

Table III: The use of chlorhexidine gel by mothers

\begin{tabular}{|c|c|}
\hline Variables & Frequency, $n=199(\%)$ \\
\hline \multicolumn{2}{|l|}{ Did you take care of last baby's cord? } \\
\hline Yes & $191(96.0)$ \\
\hline No & $8(4.0)$ \\
\hline \multicolumn{2}{|c|}{ What agent did you use for cord care in the index baby? } \\
\hline Methylated spirit only & $113(56.8)$ \\
\hline Chlorhexidine gel only & $19(9.6)$ \\
\hline Methylated spirit and chlorhexidine gel & $33(16.6)$ \\
\hline Others & $34(17.0)$ \\
\hline \multicolumn{2}{|l|}{ How soon after birth was cord care started? } \\
\hline$<$ I hour & $33(16.6)$ \\
\hline $1-23$ hours & $95(47.7)$ \\
\hline 1 day-7days & $71(35.7)$ \\
\hline \multicolumn{2}{|l|}{ Who informed the choice of cord care? } \\
\hline Antenatal clinic & $120(60.3)$ \\
\hline Other health care workers & $27(13.6)$ \\
\hline Relatives & $42(21.1)$ \\
\hline Neighbours/friends & $2(1.0)$ \\
\hline Social media & $1(0.5)$ \\
\hline Others & $7(3.5)$ \\
\hline \multicolumn{2}{|l|}{ Have you ever used chlorhexidine gel? } \\
\hline Yes & $67(33.7)$ \\
\hline No & $132(66.3)$ \\
\hline \multicolumn{2}{|c|}{ How many times did you apply chlorhexidine gel? $n=67$} \\
\hline Once & $16(23.9)$ \\
\hline More than once & $51(76.1)$ \\
\hline \multicolumn{2}{|c|}{ Did you wash your hands always before use of chlorhexidine gel? $n=67$} \\
\hline Yes & $49(73.1)$ \\
\hline No & $18(26.9)$ \\
\hline \multicolumn{2}{|l|}{ Where did you apply chlorhexidine gel? } \\
\hline Stump only & $15(22.4)$ \\
\hline Surrounding skin only & $16(23.9)$ \\
\hline Both & $36(53.7)$ \\
\hline \multicolumn{2}{|c|}{ Did you use a finger to spread chlorhexidine gel? } \\
\hline Yes & $50(74.6)$ \\
\hline No & $17(25.4)$ \\
\hline \multicolumn{2}{|c|}{ What did you do after applying chlorhexidine gel? } \\
\hline Keep cord dry & $40(59.7)$ \\
\hline Apply other agents & $27(40.3)$ \\
\hline \multicolumn{2}{|l|}{ What did you do after cord detaches? } \\
\hline Stop chlorhexidine gel application & $15(22.4)$ \\
\hline Continue for more one week & $2(3.0)$ \\
\hline Apply other agents & $50(74.6)$ \\
\hline \multicolumn{2}{|c|}{ How long did you apply chlorhexidine gel before the cord detached? } \\
\hline$<7$ days & $18(26.9)$ \\
\hline 7-14 days & $45(67.2)$ \\
\hline$>14$ days & $4(5.9)$ \\
\hline \multicolumn{2}{|c|}{ What side effects of chlorhexidine gel did you experience? $n=16$} \\
\hline Delayed cord detachment & $10(62.5)$ \\
\hline Heals poorly & $3(18.8)$ \\
\hline Bleeding & $3(18.8)$ \\
\hline
\end{tabular}

Association of Awareness, Attitude and the use of Chlorhexidine Gel

Good awareness was significantly associated with good use of CHX and vice versa, $\mathrm{P}$ value $<0.05$, Table IV.

\begin{tabular}{|} 
Table IV: Association of Awareness, Attitude and the use of Chlorhexidine Gel \\
\begin{tabular}{|ccccc|}
\hline Variables & $\begin{array}{c}\text { Practice Grades } \\
\text { Good } \\
\text { N(\%) }\end{array}$ & $\begin{array}{c}\text { Test of Association } \\
\text { Poor } \\
\mathbf{N}(\%)\end{array}$ & P value & Odds Ratio (95\% CI) \\
\hline $\begin{array}{c}\text { Knowledge Grade } \\
\text { Good } \geq 70 \%\end{array}$ & $13(72.2)$ & $55(30.4)$ & $0.001 *$ & $5.0(1.86,13.47)$ \\
Poor $<70 \%$ & $5(27.8)$ & $126(69.6)$ & & \\
Attitude Grade & $14(77.8)$ & $57(52.8)$ & 0.071 & $2.7(0.95,7.78)$ \\
Good $\geq 70 \%$ & $4(22.2)$ & $51(47.2)$ & \\
Poor $<70 \%$ & $*=$ Statistically significant \\
\hline
\end{tabular}
\end{tabular}

Association of Socio-demographic characteristics with Awareness, Attitude and the use of Chlorhexidine Gel among Mothers attending the Well-Baby Clinic

There was no statistical significance in the association of socio-demographic characteristics in respect to awareness and use scores, $\mathrm{P}$ value $>0.05$. Mother's level of 
education was significantly associated with their attitude towards the use of chlorhexidine gel, $\mathrm{P}$ value $=0.004$, Table $\mathrm{V}$.

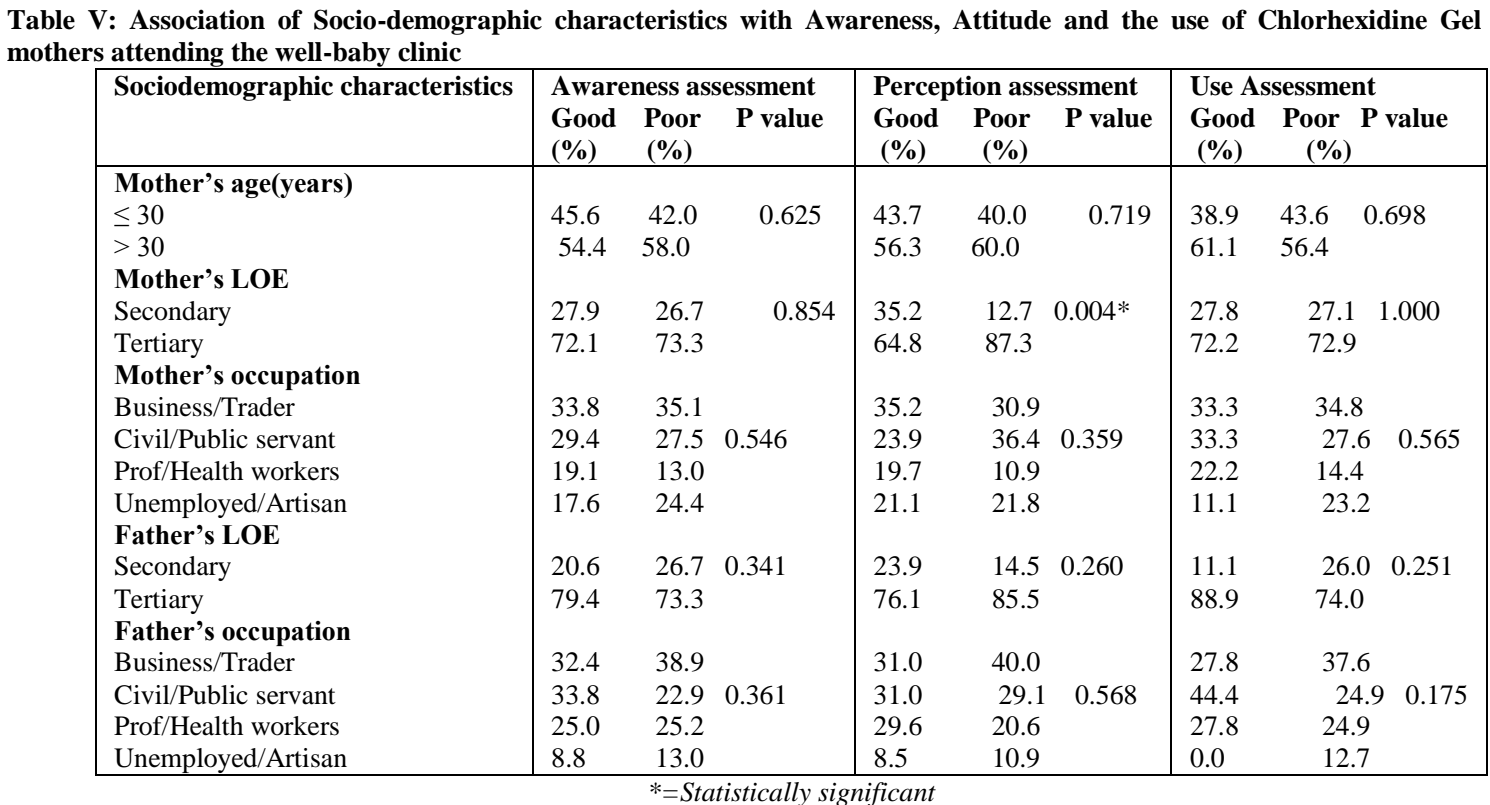

\section{DISCUSSION}

This present study shows that although most of the mothers attending ANC at RSUTH $(63.3 \%)$ had heard of chlorhexidine gel, and $100 \%$ of those who had heard knew what it was used for, only $34.4 \%$ had good knowledge of its' use. This is in contrast to the finding by Berhe et al ${ }^{20}$ where $87.3 \%$ of the study participants had good knowledge about cord care after birth. Similarly, a study done in Abeokuta ${ }^{22}$ also showed that $69 \%$ of the study subjects had good knowledge of the Use of CHX gel. This difference is probably due to the difference in the study participants. Whereas our study subjects were mothers attending ANC, health care workers were used in the other studies.

Our study showed that only $9.5 \%$ of mothers used CHX gel alone for cord care. This abysmally low proportion is similar to findings in Abeokuta ${ }^{22}$ and Ethiopia. ${ }^{20}$ In both studies, although the participants which were health care workers and as such expected to have had adequate knowledge about the recommendations for the use of CHX gel, only a few of them actually used it. This reflects the fact that many healthcare workers are yet to come to terms with the recommendations of $\mathrm{WHO}$ on the use of
CHX gel ${ }^{22}$. And as shown by the findings of our study, majority of mothers' source of information about CHX gel was from the ANC and other healthcare workers $(81.7 \%$ and $11.9 \%$ respectively). Most of the mothers also made decisions about choice of cord care agents based on information they receive during ANC and from contact with other health care workers $(60.3 \%$ and $13.6 \%$ respectively). We can therefore infer that there is inadequate communication to mothers attending ANC about information on current trends of cord care practice as recommended by the WHO.

Our study showed that only $23.9 \%$ of the mothers who used CHX gel applied it once daily as recommended by WHO. The other mothers applied CHX gel as many as 2-10 times a day. This finding is similar to the studies done in Ethiopia ${ }^{20}$ and Jos 21 which showed that most mothers applied CHX gel every 2 hours-similar to the way methylated spirit is applied. In the study done in Jos by Schwe et al ${ }^{21} \mathrm{CHX}$ gel was applied at least twice daily, to the cord and stump base as directed by the researchers. This was an experimental study where the participants were trained at the beginning of the study on how to apply the CHX gel; and the instruction according to their study 
design/protocol was to apply the CHX gel twice a day and any other time the cord appears wet. In contrast, the mothers in a study done in Kenya ${ }^{23}$, applied the CHX gel correctly-once daily. The Kenyan study involved both non-health workers and health workers as participants. Majority of the non-health worker group (16 out of 19$84 \%$ ) were mothers instructed by the researchers to apply $\mathrm{CHX}$ gel correctly-once daily. The other participants in that study were 24 health care workers and were thus more informed. The sample size was small and thus it was easier for the researchers to pass across information about proper cord care using $\mathrm{CHX}$ gel more effectively to a small group.

Our study showed that only $16.6 \%$ of the mothers who used CHX gel for cord care used it within 24 hours of child's birth. In a study done in Nepal, Mullany 24 reported about $25 \%$ reduction in mortality among newborns treated with CHX gel than in the comparison group. More so, mortality was one-third lower amongst those who received the first application within 24 hours of birth. Thus, there is a need to further emphasize early application of CHX gel (within 24 hours of birth) to the cord as recommended by WHO in order to reduce neonatal sepsis and mortality. ${ }^{13,14,15,24,25,26}$

Most mothers felt that delayed cord detachment was a side effect of $\mathrm{CHX}$ gel and over $40 \%$ were unwilling to use it again. In addition, about $60 \%$ would not recommend its use. This perception about its' inefficiency because of delay in cord detachment may explain why majority of the mothers in our study kept on applying other agents even after the cord stump fell off (74.6\%). Cultural beliefs of communities influence a mother's perception of how long it should take for the cord to fall off. This explains why some of the mothers in our study applied other agents after some days of using CHX gel, to hasten drying of the cord. This is similar to the practice of some mothers reported in the study by Asiedu in Ghana. ${ }^{10}$ The study by Ambale et al in Kenya ${ }^{23}$ also reported that some mothers used other agents like spirit, warm water, normal saline and antiseptics to clean off the dry flakes left by the gel on the cord. Other side effects reported in our study included bleeding from the cord stump in 3 babies and poor stump healing in 3 other babies. Schwe et al ${ }^{21}$ in Jos reported bullous eruptions on anterior abdominal wall in onetenth (3 out of 30 ) of the study subjects in the group who used CHX gel for cord care.

Our study showed majority of mothers $(73,4 \%)$ used spirit either alone (56.8\%), or in combination with $\mathrm{CHX}$ gel $(16.6 \%)$. Of the $9.5 \%$ of mothers who used only CHX gel for cord care, $58.7 \%$ believe it's effective but only $49.2 \%$ said they will use it again. Similarly, the study done in Kenya ${ }^{23}$ among some mothers and health workers in Kangundo hospital showed both mothers and health workers felt that methylated spirit was more or equally effective as a cord care agent. Less than $25 \%$ of both groups in the study felt $\mathrm{CHX}$ gel was more effective. It seems therefore across both groups-health workers and mothers alike, the perception is that methylated spirit is more effective as the cord separation time reported was shorter with the use of methylated spirit. In contrast to our findings, the Jos study ${ }^{21}$, reports shorter cord separation time with the use of CHX gel. This could be because of the design of the study as mothers were properly instructed on how to use CHX gel prior to commencement of the study. The mothers in our study received no prior instructions and applied the gel based on their own understanding.

Agents used for cord care by mothers recruited in our study included CHX gel, methylated spirit, hot water compress, toothpaste, salt, powder, mentholatum, petroleum jelly, antiseptics/disinfectant, penicillin and native herbs. Similarly, in Ghana, Asiedu et al ${ }^{10}$ reported the use of agents such as toothpaste, salt, sand, petroleum jelly, shea butter, herbs, chalk, ground banana peels, antiseptics like Dettol, baby lotion, penicillin and amoxicillin. This similarity is 
probably due to the fact that both studies were conducted in West African countries (Nigeria and Ghana) with similar cultures. Hodgins et al ${ }^{26}$ reported the application of spices, butter, oil, ash and mud for cord care in rural India and Nepal. The various agents used vary from culture to culture.

Our study showed good awareness was significantly associated with good use of CHX gel. This is not surprising as studies have shown that with increased awareness following robust public health campaigns, the use of CHX gel was better with a resultant reduction in neonatal mortality/ morbidity. ${ }^{16,17,20,26,27,30}$ Also the studies done in Jos ${ }^{21}$ and Kenya ${ }^{23}$ also showed better use/practice because the mothers received prior instructions in even smaller groups.

Similarly, our study showed that the mothers with tertiary level of education significantly had better attitude towards using CHX gel. Similarly, the study done by Asiedu et al ${ }^{28}$ in Ghana also showed the mothers level of education was associated with the type of dressing used for cord care. Mothers with no formal education were found to use unapproved substances such as chalk, salt, toothpaste and sand. Similarly, Abhulimhen-Iyoha and Ibadin ${ }^{29}$ in Benin city found that mothers who had attained at least primary level of education were more likely to use approved dressings such as methylated spirit whereas those with no formal education were more likely to use unapproved dressings for cord care. ${ }^{28,29}$

The recommendation by WHO for the use of CHX gel for newborn cord care in a country with high risk for NNS, such as ours, needs to be reemphasized as this will lead to a reduction in neonatal mortality rate. ${ }^{26,27,30}$

\section{CONCLUSION}

Our study shows awareness, attitude and the use of CHX gel for cord care by mothers in Port Harcourt is still relatively low. The need to increase awareness about CHX gel, it's proper use and benefits; by massive public health campaign strategies, training and retraining of health care workers especially midwives involved in health instruction during ANC, CHEWs, TBAs, religious and community leaders and the general public at large, cannot be over emphasized. Government policies should be strengthened to support the scale-up of its' use not just in some Northern states but rather all over the country.

\section{Limitations}

Our study was based on recall and therefore there could be recall bias.

\section{Acknowledgement: None}

\section{Conflict of Interest: None}

\section{Source of Funding: None}

\section{Ethical Approval: Approved}

\section{REFERENCES}

1. Draiko CV, McKague K, Maturu JD, Sitima J. The effect of umbilical cord cleansing with chlorhexidine gel on neonatal mortality among the community births in South Sudan: a quasi-experimental study. Pan African Medical Journal. 2021;38(78). 10.11604/pamj.2021.38.78.21713

2. National Population Commission (NPC) [Nigeria] and ICF. 2019. Nigeria Demographic and Health Survey 2018.Abuja, Nigeria, and Rockville, Maryland, USA: NPC and ICF.

3. Gallagher PG, Shah SS, Windle ML, Carter BS. Omphalitis, 2019[Internet\}. Available from:https://emedicine.medscape.com/articl e/975422-overview.Assessed 30/4/2021

4. World Health Organization. WHO Recommendations on Postnatal Care of the Mother and Newborn. Geneva, Switzerland: WHO Press; 2014

5. Stewart D, Benitz W. AAP COMMITTEE ON FETUS AND NEWBORN. Umbilical Cord Care in the Newborn Infant. Pediatrics. 2016;138(3):2016-2149. DOI: $10.1542 /$ peds.

6. Herlihy JM, Shaikh A, Mazimba A, Gagne N, Grogan C, Mpamba C, Sooli B, Simamvwa G, Mabeta C, Shankoti P, et al. Local perceptions, cultural beliefs and practices that shape umbilical cord care: a 
qualitative study in Southern Province, Zambia. PLoS One. 2013;8(11): e79191.

7. Sacks E, Moss WJ, Winch PJ, Thuma P, van Dijk JH, Mullany LC. Skin, thermal and umbilical cord care practices for neonates in southern, rural Zambia: a qualitative study. BMC Pregnancy Childbirth. 2015; 15:149.

8. Semrau KE, Herlihy J, Grogan C, Musokotwane K, Yeboah-Antwi K, Mbewe R, Banda B, Mpamba C, Hamomba F, Pilingana $\mathrm{P}$, et al. Effectiveness of $4 \%$ chlorhexidine umbilical cord care on neonatal mortality in Southern Province, Zambia (ZamCAT): a cluster-randomized controlled trial. Lancet Glob Health. 2016;4(11):827-36.

9. Opara PI, Jaja T, Okari TG. Newborn cord care practices among mothers in Port Harcourt, Niger Jos J Med, 2016; 6(3):3236.

10. Opoku Asiedu SS, Apatu NAA, Tetteh R, Hodgson A. Neonatal cord care practices among mothers and caregivers in the Volta Region of Ghana. Int J MCH AIDS. 2019; 8(1):63-69. DOI: 10.21106/ijma.272

11. World Health Organization. World Health organization recommendations on postnatal care of the mother and newborn 2013. Available from http://apps.who.int/iris/bitstream/10665/976 03/1/9789241506649_eng.pdf Assessed on $4 / 5 / 21$

12. Chlorhexidine Working Group. Chlorhexidine for umbilical cord care: A new, low-cost intervention to reduce newborn mortality. Available at http://www.healthynewbornnetwork.org/iss ue/chlorhexidine-for-umbilical-cord-care/ Assessed 4/5/21

13. Sinha A, Sazawal S, Pradhan A, Ramji S, Opiyo N. Chlorhexidine skin or cord care for prevention of mortality and infections in neonates. Cochrane Database Syst Rev. 2015;3:CD007835

14. Arifeen SE, Mullany LC, Shah R, et al. The effect of cord cleansing with chlorhexidine on neonatal mortality in rural Bangladesh: a community-based, cluster-randomised trial. Lancet. 2012;379(9820):1022-28

15. Soofi S, Cousens S, Imdad A, Bhutto N, Ali $\mathrm{N}$, Bhutta ZA. Topical application of chlorhexidine to neonatal umbilical cords for prevention of omphalitis and neonatal mortality in a rural district of Pakistan: a community-based, cluster-randomised trial. Lancet. 2012;379(9820):1029-36

16. Federal Ministry of Health, USAID. Scaling up Chlorhexidine for umbilical cord care in Nigeria. FMOH, USAID, Abuja 2016.

17. Orobaton N, Abegunde D, Shoretire K, Abdulazeez J, Fapohunda B, Lamiri G, et al. (2015) A Report of At-Scale Distribution of Chlorhexidine Digluconate $7.1 \%$ Gel for Newborn Cord Care to 36,404 Newborns in Sokoto State, Nigeria: Initial Lessons Learned. PLoS ONE 10(7): e0134040. DOI: 10.1371/journal.pone. 0134040

18. Abegunde D, Orobaton N, Beal K, Bassi A, Bamidele M, Akomolafe $\mathrm{T}$ et al. Trends in newborn umbilical cord care practices in Sokoto and Bauchi States of Nigeria: the where, who, how, what and the ubiquitous role of traditional birth attendants: a lot quality assurance sampling survey. BMC Preg Childbirth. 2017; 17:368 DOI: 10.1186/s12884-017-1551-x

19. Mohammed A, Envuladu EA, Osagie IA, Difa JA. Assessment of knowledge and cord care practices among pregnant women in selected PHCs in Jos metropolis, Plateau state. Int J Community Med Public Health. 2020; 7:1215-9.

20. Berhe AK, Tinsae F, Gebreegziabher G. Knowledge and practice of immediate newborn care among health care providers in eastern zone public health facilities, Tigray, Ethopia. BMC Paediatrics 2017; 17: 157. DOI 10.1186/s12887-017-0915-8

21. Shwe DD, Abok II, Diala UM, Egbodo C, Toma BO, Nathan S, Pam VC, Ocheke A, Ogwuche S. Methylated spirit versus $4 \%$ chlorhexidine gel in neonatal umbilical cord infection: A short report of a randomized, open-labelled, parallel-group trial. Niger $\mathrm{j}$ Paediatr. 2018; 45 (2): 118-122

22. Ikperite U, Ope-Babadele O, Ojo EA. Awareness and Use of Chlorhexidene Gel in Prevention of Neonatal sepsis among Nurses and Midwives in selected Health Facilities in Abeokuta, Nigeria. Saudi J Nurs Health Care. 2020; 3(2): 28-31.

23. Ambale C, Ngathia B, Nthusi J, Mukonzo J. Assessment of the use of Chlorhexidene Digluconate Gel for cord Care at Kangundo level 4 Hospital. International Journal of Scientific and Research Publications, Volume 11, issue 1, January 2021. DOI: 10.29322/IJSRP.11.01.2021. p10932. 
24. Mullany LC, Darmstadt GL, Khatry SK, Katz J, Leclerq SC, Shrestha S, et al. Topical applications of chlorhexidine to the umbilical cord for prevention of omphalitis and neonatal mortality in southern Nepal: a community-based, cluster-randomized trial. Lancet. 2006; 367(9514):910-18. CrossRef. Medline

25. World health Organisation(WHO). Care of the umbilical cord(Internet). Geneva: WHO; 1999.Cited 2012 Jul 1. Available from: https://apps.who.int/rht/documents/MSM984/MSM-98-4,htm

26. Hodgins S, Pradhan YV, Khanal L, Upreti S, Pratap NKC. Chlorhexidene for umbilical cord care: game-changer for newborn survival? Global Health: Science and Practice 2013. Volume 1 Number 1.

27. Sankar MJ, chandrasekaran A, Ravindranath A, Agarwal R, Paul VK. Umbilical Cord cleansing with chlorhexidine in neonates: a systematic review. Journal of Perinatology. 2016; $\quad 36, \quad$ S12-S20; $\quad$ DOI: $10.1038 /$ jp. 2016.28
28. Asiedu SSO, Apatu NAA, Tetteh R, Hodgson A. Neonatal Cord Care Practices among Mothers and Caregivers in the Volta Region of Ghana. International Journal of MCH and AIDS. 2019; Vol. 8, No 1. 63-69.

29. Abhulimhen-Iyoha B, Ibadin M. Determinants of cord care practices among mothers in Benin City, Edo state, Nigeria. Niger J Clin Pract. 2012; 15(2): 210. DOI: 10.4103/1119-3077.97320.

30. Gelano TF, Bacha YD, Abate D. Effect of chlorhexidine cord application on prevention of neonatal sepsis in developing countries: Systematic review and metaanalysis. Int J Health Sci (Qassim). 2019 Jan-Feb; 13(1): 40-51.

How to cite this article: Aitafo JE, West BA, Okari TG. Awareness, attitude and use of chlorhexidine gel for cord care in a well-baby clinic in Port Harcourt, Nigeria. Int J Health Sci Res. 2021; 11(8): 180-189. DOI: https://doi. org/10.52403/ijhsr.20210826 